九州大学学術情報リポジトリ

Kyushu University Institutional Repository

Effect of Nanoparticle Concentration on the Specific Heat Capacity and Thermal Stability of Graphite Nanoparticle-based Molten Salt

Nusrat Jahan Salim

Department of Mechanical Engineering, Bangladesh University of Engineering and Technology

Md. Ashiqur Rahman

Department of Mechanical Engineering, Bangladesh University of Engineering and Technology

https://doi.org/10.5109/4102463

出版情報: Proceedings of International Exchange and Innovation Conference on Engineering \& Sciences (IEICES). 6, pp.52-59, 2020-10-22. Interdisciplinary Graduate School of Engineering Sciences, Kyushu University

バージョン：

権利関係 : 


\title{
Effect of Nanoparticle Concentration on the Specific Heat Capacity and Thermal Stability of Graphite Nanoparticle-based Molten Salt
}

\author{
Nusrat Jahan Salim, Md. Ashiqur Rahman \\ Department of Mechanical Engineering, Bangladesh University of Engineering and Technology, Dhaka, Bangladesh. \\ Corresponding author email: nusratsalim@me.buet.ac.bd
}

\begin{abstract}
This study targets at examining the effect of nanoparticle concentration on the specific heat capacity $\left(C_{p}\right)$ and thermal stability of binary carbonate salt eutectic mixture (lithium carbonate and potassium carbonate at a molar ratio of 62:38, respectively) doped with three different weight percentages (2\%, 4\%, and 6\%) of graphite nanoparticles $(G N P)$. Simultaneous thermal analyzer (STA) is used to obtain the $C_{p}$ and thermal stability of the prepared samples in both solid and liquid phases. The experimental findings show that the increasing mass concentration of graphite nanoparticles can positively affect the performance of the nanocomposites in thermal energy storage (TES) system. The highest specific heat capacity enhancement of $25 \%$ is observed in case of $6 \%$ (wt.) graphite nanoparticle inclusion in liquid phase. The superior thermal properties of the nanocomposites and mass concentration-controlled $C_{p}$ enhancement are explained in regard to the formation of compressed layers on nanoparticle surface and particle agglomeration tendency.
\end{abstract}

Keywords: Binary carbonate salt; Graphite nanoparticles; Specific heat; Thermal stability; Compressed layer.

\section{INTRODUCTION}

Researchers all over the world are actively working towards the efficient harnessing of widely abundant solar energy due to its obvious importance in achieving clean and sustainable energy in the future [1]. But due to the intermittent nature of this form of renewable energy, storage media like storage batteries and TES systems in concentrated solar power (CSP) plants are necessary for the uninterrupted use of solar energy [2]. For achieving high thermodynamic efficiency in CSP plants, molten salt eutectics are employed as phase change materials (PCMs) for TES as they possess high thermal conductivity and high density [3]. Among various molten salt eutectics binary carbonate salt eutectic mixture, which consists of lithium carbonate $\left(\mathrm{Li}_{2} \mathrm{CO}_{3}\right)$ and potassium carbonate $\left(\mathrm{K}_{2} \mathrm{CO}_{3}\right)$, at a molar ratio of $62: 38$ respectively, possesses a high melting point and melting enthalpy ensuring the higher operating temperature of CSP plants [4]. But binary carbonate salt eutectic mixture has low specific heat capacity, which is an impediment to enhance the efficiency of CSP power plants [4].

With the improvement of technology, different nanoparticle synthesis techniques have been developed. Nanoparticles often exhibit unexpected properties [5], and due to large surface to volume ratio, they obtain enormous driving force to diffuse [6]. This is a valuable property that can positively affect the thermal properties while these particles are incorporated with any base material. In 2003, a report of drastic enhancement of critical heat flux in pool boiling of nanofluids encouraged researchers to explore the possibility and mechanism to enhance the thermal properties (thermal conductivity, specific heat capacity) of heat transfer fluids (HTFs) by the dispersion of nanoparticles. Recent review papers elucidate the measurement procedure, theoretical models, and factors affecting the thermal conductivity of nanofluids [7-8]. On the contrary, the specific heat capacity of nanofluids has not been investigated extensively. However, Zhou et al. reported a decrease in specific heat capacity with increasing nanoparticle volume fraction for $\mathrm{Al}_{2} \mathrm{O}_{3}$-water-based nanofluid, which was in complete agreement with the thermal equilibrium model [9]. Akilu et al. reported the decrement in specific heat capacities of $\mathrm{SiO}_{2}-$ Glycerol, $\mathrm{SiO}_{2}-$ Ethylene Glycol, and $\mathrm{SiO}_{2}-$ Glycerol/Ethylene based nanofluids [10]. The rate of decrement depended upon the type of base fluid and this study also was in satisfactory agreement with the thermal equilibrium model. However, previous reports also suggested enhanced specific heat capacity upon the loading of nanoparticles with base fluids. Shin and Banerjee showed a $14.5 \%$ increment in specific heat capacity of chloride salt while doped with 1 wt.\% silica nanoparticle [11]. Jo and Banerjee employed four different concentrations $(0.1,0.5,1$, and 5 wt.\%) of carbon nanotube with binary carbonate salt eutectic mixture and reported drastic enhancement in specific heat capacity in each case both in the solid and liquid phases [12]. Another study of Jo and Banerjee showed up to $21 \%$ increment in $\mathrm{C}_{\mathrm{p}}$ of binary carbonate molten salts using graphite nanoparticles (1 wt.\%) and $17 \%$ increment for carbon nanotube-based (1 wt.\%) nanofluid [13]. Tiznobaik and Shin measured enhancement of $C_{p}$ of binary carbonate salt eutectic up to $29 \%$ upon loading of 1 wt. $\% \mathrm{SiO}_{2}$ [14]. Jo and Banerjee reported up to $100 \%$ increment in $\mathrm{Cp}$ of the same kind of salt by including 1 wt.\% graphite nanoparticles [15]. Another study of Kim and Jo reported a more than $30 \%$ increase in binary carbonate molten salt mixture in liquid phase with $1 \mathrm{wt} . \%$ graphite nanoparticle inclusion [16]. All these increments mentioned above were drastic and the patterns did not match well with the thermal equilibrium model - which takes into account the type of base material and nanomaterial as the deciding parameters for $C_{p}$ enhancement. Extensive studies have been carried out by Dr. Banerjee's research group which showed that specific heat capacity increment depends on several parameters other than the type of base material and nanomaterials, like - type of dispersant used in the preparation process, dispersion homogeneity, evaporation duration, and evaporation temperature $[12,17]$. The numerical and experimental study also found the role of compressed 
layers behind the drastic enhancement of specific heat capacity $[15,18]$.

As mentioned above, there exists controversy between theoretical prediction and experimental findings of specific heat capacity - extreme increment in specific heat capacity cannot be predicted with the existing thermal equilibrium model. Moreover, the effect of nanoparticle concentration on the formation of compressed layers has not been sufficiently discussed for any type of nanoparticle-based molten salts. Previous studies were mostly limited to $1 \mathrm{wt} \%$ nanoparticle dispersion in binary carbonate molten salts and thus the effect of nanoparticle concentration on compressed liquid layer formation has not been elucidated yet.

This paper aims to examine the influence of nanoparticle concentrations on the specific heat capacity and thermal stability of the molten salt. The properties were investigated for three different concentrations $(2,4,6$ wt.\%) of GNPs dispersed in the binary carbonate salt eutectic mixture. Comparison of the pure eutectic mixture with nanomaterials was done both in the solid phase $\left(250^{\circ} \mathrm{C}\right.$ and $\left.400^{\circ} \mathrm{C}\right)$ and the liquid phase $\left(520^{\circ} \mathrm{C}\right.$ $560^{\circ} \mathrm{C}$ ). The SEM micrographs were obtained for preSTA samples to ensure the nanoparticle dispersion and observe the change in compressed layer formation. The thermal stability of the samples with thermal cycles and high-temperature conditions were tested. Finally, the reason behind the discrepancy of the specific heat capacity between the experimentally measured values in the present study and theoretical predictions by the thermal equilibrium model was also explained.

\section{MATERIALS AND METHODS}

\subsection{Preparation of molten salt nanomaterials}

Binary carbonate salt eutectic mixture was used as the base material in this experiment and graphite nanoparticles at three different mass ratios $(2 \%, 4 \%$, and $6 \%$ ) were incorporated with the base material. $\mathrm{Li}_{2} \mathrm{CO}_{3}+$ $\mathrm{K}_{2} \mathrm{CO}_{3}$ (62:38 molar ratio) was procured from the local market, graphite nanoparticles were procured from US Research Nanomaterials, Inc. The average particle size was $400 \mathrm{~nm}$ according to the manufacturer specification. All these materials were used without further refinement. The synthesis procedure of the binary carbonate salt eutectic mixture-based graphite nanomaterials is shown in Fig. 1. All synthesis protocols developed by research group of Banerjee was followed during the nanomaterial preparation [11-12,17,19].

First, GNPs of required mass were dispersed in distilled water with gum arabic (GA) at a mass concentration of $1 \%$ with respect to the eutectic to avoid agglomeration of the GNPs. This colloidal suspension was sonicated in an

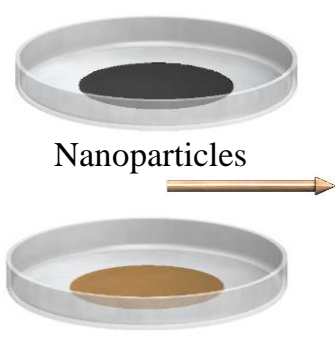

Gum arabic

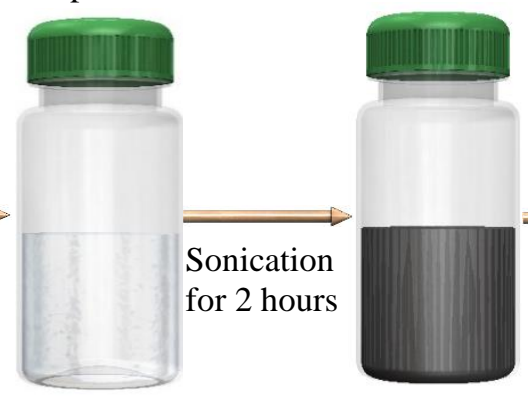

Distilled water ultrasonic bath for two hours. Then additional water with the eutectic mixture was added to the colloid and again subjected to sonication for three additional hours. The obtained suspension was poured into a petri dish with a large diameter to shorten the evaporation time and maintain the homogeneity of the sample. Finally, the petri dish was placed on a hot plate and water was evaporated at $100^{\circ} \mathrm{C}$ to obtain the dry nanomaterial sample.

\subsection{Measurement of specific heat capacity and thermal stability}

The specific heat capacity $\left(C_{p}\right)$ and thermal stability of all the samples (molten salt eutectic and nanomaterials) were measured with simultaneous thermal analyzer (STA 449 F3 Jupiter). The standard test method (ASTM E1269 - 11) was followed to determine the specific heat capacity of the materials over a wide temperature range $\left(150^{\circ} \mathrm{C}-560^{\circ} \mathrm{C}\right)[20]$. This temperature range presents the $\mathrm{C}_{\mathrm{p}}$ of both solid phase and liquid phase $\left(520^{\circ} \mathrm{C}-560^{\circ} \mathrm{C}\right)$ of the molten salt and nanomaterials. For the convenience of our discussion, we will mention the terms "nanocomposite" for the solid phase and "nanofluid" for the liquid phase. First, the STA apparatus was purged with dry nitrogen at a flow rate of $40 \mathrm{~mL} / \mathrm{min}$ and it was maintained throughout the experiment. The weight of the empty alumina pan (specimen holder) with lid was recorded as the tare weight. The empty specimen holder plus lid and a reference specimen holder plus lid were positioned in the apparatus. The following experimental conditions were set to obtain the differential scanning calorimeter (DSC) and thermogravimetric analysis (TGA) graphs - isothermal temperatures (at $150^{\circ} \mathrm{C}$ for 5 minutes and $560^{\circ} \mathrm{C}$ for 5 minutes) and ramp rate $\left(20^{\circ} \mathrm{C} / \mathrm{min}\right)$. This step is called the baseline test according to the standard protocol. In the next step (reference test), a reference material (sapphire) was kept in the empty pan and the above-mentioned steps were followed again. Finally, the sample test was done following the same steps with the sample material in the empty pan. Each of these steps was repeated five times for each sample for the accuracy and reproducibility of the experimental results. Data from the first cycle was discarded due to the skewness. Experimental specific heat capacity was measured from the data obtained from these three steps [21]. Fig. 2 shows the DSC heat flow curve for two different samples and a marginal increase in the heat absorption was observed from the inset plot for the nanomaterial samples compared with the pure eutectic mixture. Though the inset plot exhibits marginal increase in heat flow within a small temperature range, the pattern

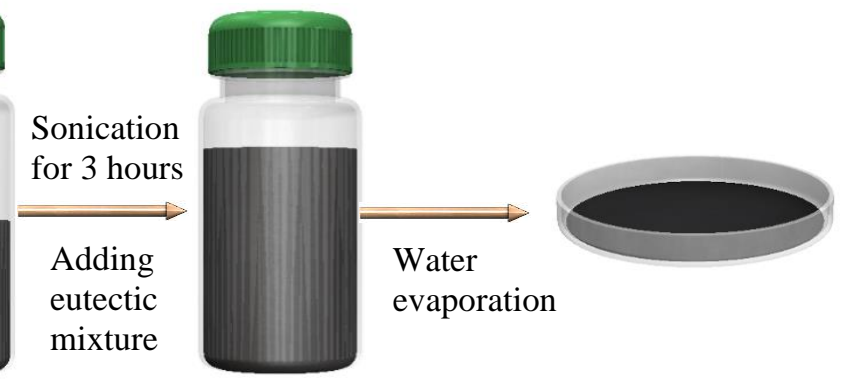

Fig. 1 Preparation of eutectic mixture-based nanomaterial. 
$\frac{U\left(C_{s}\right)}{C_{s}}=\sqrt{\left(\frac{U\left(C_{s t}\right)}{C_{s t}}\right)^{2}+\left(\frac{U\left(q_{b}\right)}{q_{b}}\right)^{2}+\left(\frac{U\left(q_{s}\right)}{q_{s}}\right)^{2}+\left(\frac{U\left(q_{s t}\right)}{q_{s t}}\right)^{2}+\left(\frac{U\left(m_{s}\right)}{m_{s}}\right)^{2}+\left(\frac{U\left(m_{s t}\right)}{m_{s t}}\right)^{2}}$

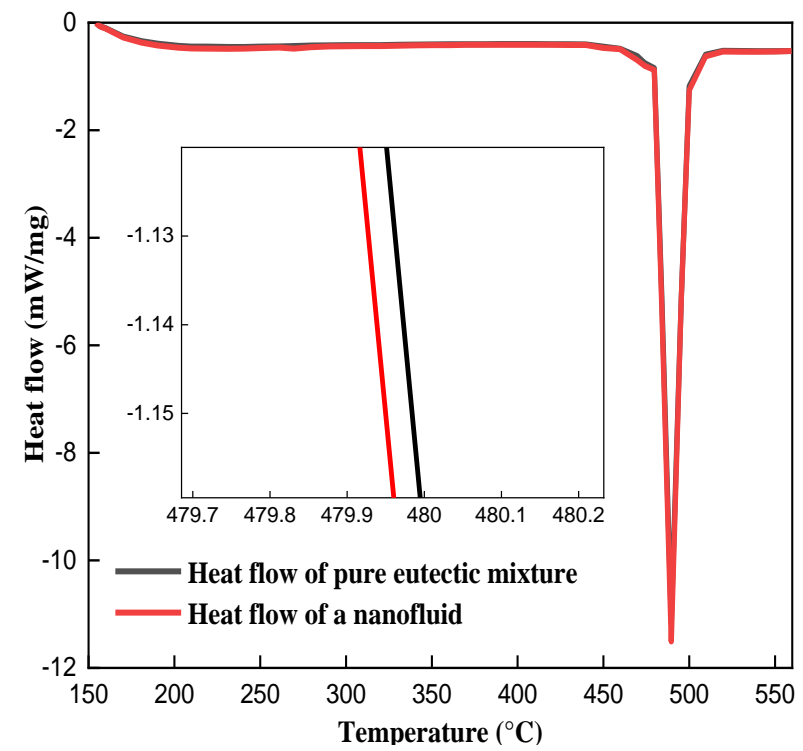

Fig. 2 Variation of DSC endothermic heat flow curve with temperature for pure eutectic mixture and a nanomaterial.

$\left(150^{\circ}-560^{\circ} \mathrm{C}\right)$.

As the measurement was carried out in STA, a precision balance was employed with the disk to continuously measure the mass change, which occurs due to phase transition, chemical reaction, loss of vapor content, etc. The temperature was increased at a constant rate to observe any mass change due to thermal reaction. Data of remaining mass percentage with respect to the initial mass as a function of temperature change was obtained from the STA.

The thermal equilibrium model (Eqn. 1) was employed to theoretically predict the $\mathrm{C}_{\mathrm{p}}$ of nanocomposites and nanofluids. This model was first proposed by Buongiorno et al. [22] and since then it was used by many researchers to predict the specific heat capacity of nanofluids as well as nanocomposites $[8,11]$.

$$
C_{p, n f}=\frac{m_{n} \cdot C_{p, n}+m_{f} \cdot C_{p, f}}{m_{n}+m_{f}}
$$

Where, $C_{p, n f}, C_{p, n}, C_{p, f}, m_{n}, m_{f}$ denote the specific heat capacity of nanofluid, specific heat capacity of the nanoparticle, specific heat capacity of molten salt, mass fraction of the nanoparticle, and mass fraction of the molten salt respectively.

\subsection{Measurement of uncertainty}

Specific heat capacity of the nanocomposite and nanofluid from experimental data were obtained from the following equation according to the ASTM standard,

$$
C_{p, s}=C_{p, s t} \frac{\Lambda q_{s} \cdot m_{s t}}{\underline{\Lambda} q_{s t} \cdot m_{s}}
$$

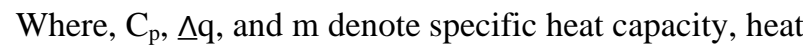
flow difference between empty pan and specimen, and mass, respectively. Subscript s and st stand for sample and standard material, respectively. Thus, some measurement.

The uncertainty analysis was done with the help of the following equation [23]:

The uncertainty occurred from the specific heat capacity curve of sapphire, heat flow difference between sample side and specimen side, and mass of the specimen. Combining all these results, the uncertainty found in our experiment was $\pm 3.5 \%$.

\section{RESULTS}

3.1 Specific heat capacity obtained from experiment The theoretical value of $\mathrm{C}_{\mathrm{p}}$ for the pure eutectic mixture at $250^{\circ} \mathrm{C}$, obtained from the following equation [24] is $1.28 \mathrm{~J} / \mathrm{g}-\mathrm{K}$.

$$
\begin{aligned}
& \left.\mathrm{C}_{\mathrm{p}}=0.562+1.16 \times 10^{-3} \mathrm{~T}(\mathrm{~J} / \mathrm{g}-\mathrm{K}) \quad \text { (Eqn. } 4\right) \\
& (365 \mathrm{~K} \leq \mathrm{T} \leq 632 \mathrm{~K})
\end{aligned}
$$

In the present study, the experimental value of $\mathrm{C}_{\mathrm{p}}$ at $250^{\circ} \mathrm{C}$ for the pure eutectic mixture was $1.25 \mathrm{~J} / \mathrm{g}-\mathrm{K}$. As we have an uncertainty of measurement of $3.46 \%$, this small discrepancy is in an acceptable limit. Fig. 3 (a-b) shows the experimentally found $\mathrm{C}_{\mathrm{p}}$ values of all the nanocomposites as a function of GNP concentration and Fig. 3(d) illustrates the enhancement in $C_{p}$ of the prepared nanosalts compared to the pure eutectic mixture. The obtained specific heat capacity of binary carbonate salt eutectic mixture is in good agreement with previously reported values [12].

$\mathrm{C}_{\mathrm{p}}$ increased with increasing temperature as well as increasing mass concentration of nanoparticles. The lowest enhancement in solid phase was observed at $250^{\circ} \mathrm{C}$ with 2 wt.\% GNP inclusion (11.5\%) and the highest enhancement in solid phase was about $24 \%$ for nanoparticle concentration of 6 wt. $\%$ at $400^{\circ} \mathrm{C}$. Fig. 3(c) illustrates the $C_{p}$ values of nanofluid with varying nanoparticle concentration.

A higher enhancement (about 25\%) was obtained in liquid phase compared to the increment of $\mathrm{C}_{\mathrm{p}}$ in solid phase. The sample with the highest value of $\mathrm{C}_{\mathrm{p}}(2.05 \mathrm{~J} / \mathrm{g}$ K) consisted of $6 \mathrm{wt}$ \% GNPs in liquid phase. Similar to the solid phase, specific heat capacity enhanced with enhancing mass concentration of nanoparticles in molten state. Thus, Specific heat capacity enhanced with increasing mass concentration and temperature. However, the rate of increment slowed down at a higher mass concentration with increasing temperature as can be seen from Fig. 3(d). This is an indication that further increment in the mass concentration of nanoparticles may result in getting a threshold point.

\subsection{Theoretical prediction of specific heat capacity}

Three specific temperatures were selected to predict the theoretical values of $\mathrm{C}_{\mathrm{p}}$ of all the prepared samples using Eqn. 1. The specific heat capacity of graphite and eutectic mixture, which were employed to derive the theoretical specific heat capacity of all the samples are shown in table 1. Fig. 4 shows the pattern of specific heat capacity enhancement with respect to the pure molten salt as a 
function of temperature and nanoparticle concentration. $\mathrm{C}_{\mathrm{p}}$ increased with the nanoparticle concentration but unlike the experimentally obtained values, theoretical specific heat capacity showed a higher increment at
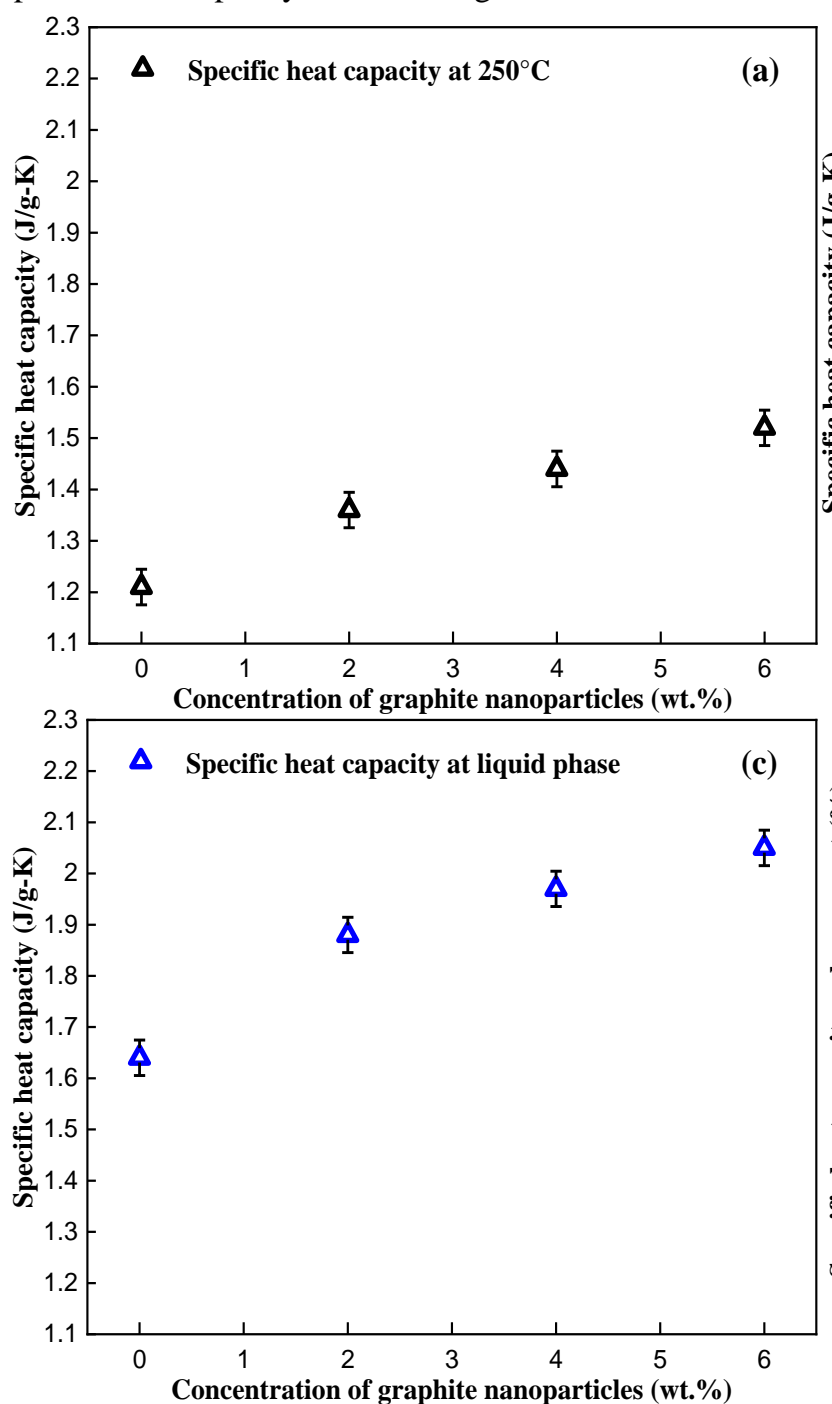

mixture and the nanocomposites for two thermal cycles (first and fifth thermal cycles). The curve from the first thermal cycle for every sample showed different amounts of mass loss up to $100^{\circ} \mathrm{C}$. Mass loss within this
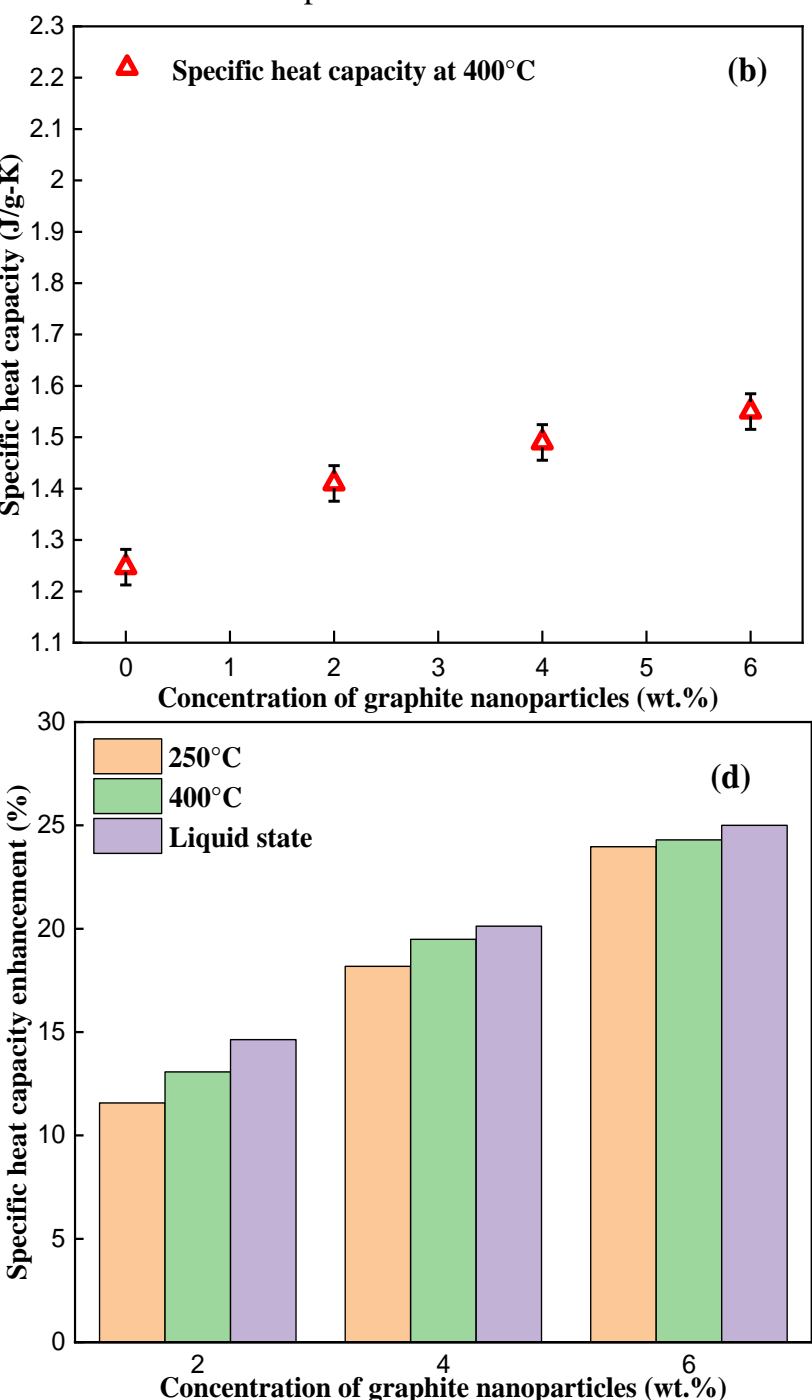

Fig. 3 Experimentally obtained specific heat capacity of the prepared samples at (a) $250^{\circ} \mathrm{C}$, (b) $400^{\circ} \mathrm{C}$, (c) liquid phase $\left(520^{\circ} \mathrm{C}-560^{\circ} \mathrm{C}\right)$ as a function of GNP concentration, and (d) enhancement of specific heat capacity of nanomaterials with respect to the pure eutectic mixture as a function of GNP concentration at different temperatures.

Table 1 . Specific heat capacity of binary carbonate salt eutectic mixture and graphite

\begin{tabular}{lll}
\hline \hline $\begin{array}{l}\text { Temperature } \\
\left({ }^{\circ} \mathrm{C}\right)\end{array}$ & $\begin{array}{l}\text { Binary } \\
\text { carobonate } \\
\text { eutectic }(\mathrm{J} / \mathrm{g}-\mathrm{K})\end{array}$ & $\begin{array}{l}\text { Graphite }(\mathrm{J} / \mathrm{g}-\mathrm{K}) \\
{[25]}\end{array}$ \\
\hline 250 & 1.21 & 1.25 \\
400 & 1.25 & 1.50 \\
$520-560$ & 1.64 & 1.66 \\
& & \\
\hline \hline
\end{tabular}

$400^{\circ} \mathrm{C}$ and the highest $\mathrm{C}_{\mathrm{p}}$ was obtained for the inclusion of $6 \%$ mass concentration of graphite at $400^{\circ} \mathrm{C}$. However, the enhancement is negligible (about $1.2 \%$ ) compared to the experimentally obtained result (24\%). Moreover, the lowest increment is observed in $520^{\circ} \mathrm{C}-560^{\circ} \mathrm{C}$ at all concentrations, which contradicts the practical values.

\subsection{Thermal stability analysis}

The thermogravimetric analysis of the molten salts under $\mathrm{N}_{2}$ environment gave us insights into the thermal stability of the prepared salts. Fig. 5 shows the TGA curve (temperature range: $25^{\circ} \mathrm{C}-560^{\circ} \mathrm{C}$ ) of the pure eutectic temperature range occurs due to the loss of moisture content which retains within the material after

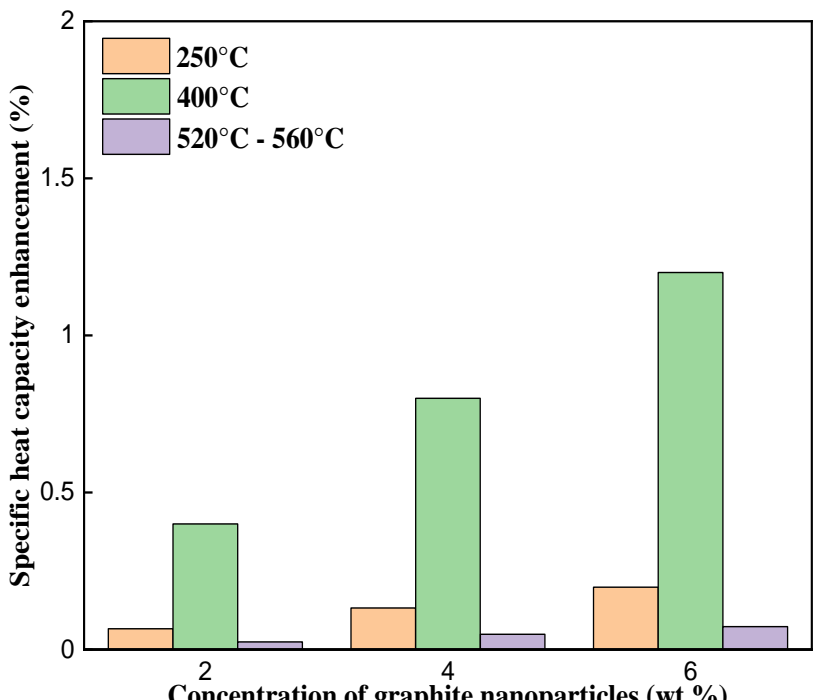

Fig. 4 Theoretical enhancement in specific heat capacity for the nanoparticle-based molten salts compared to the pure eutectic mixture. 

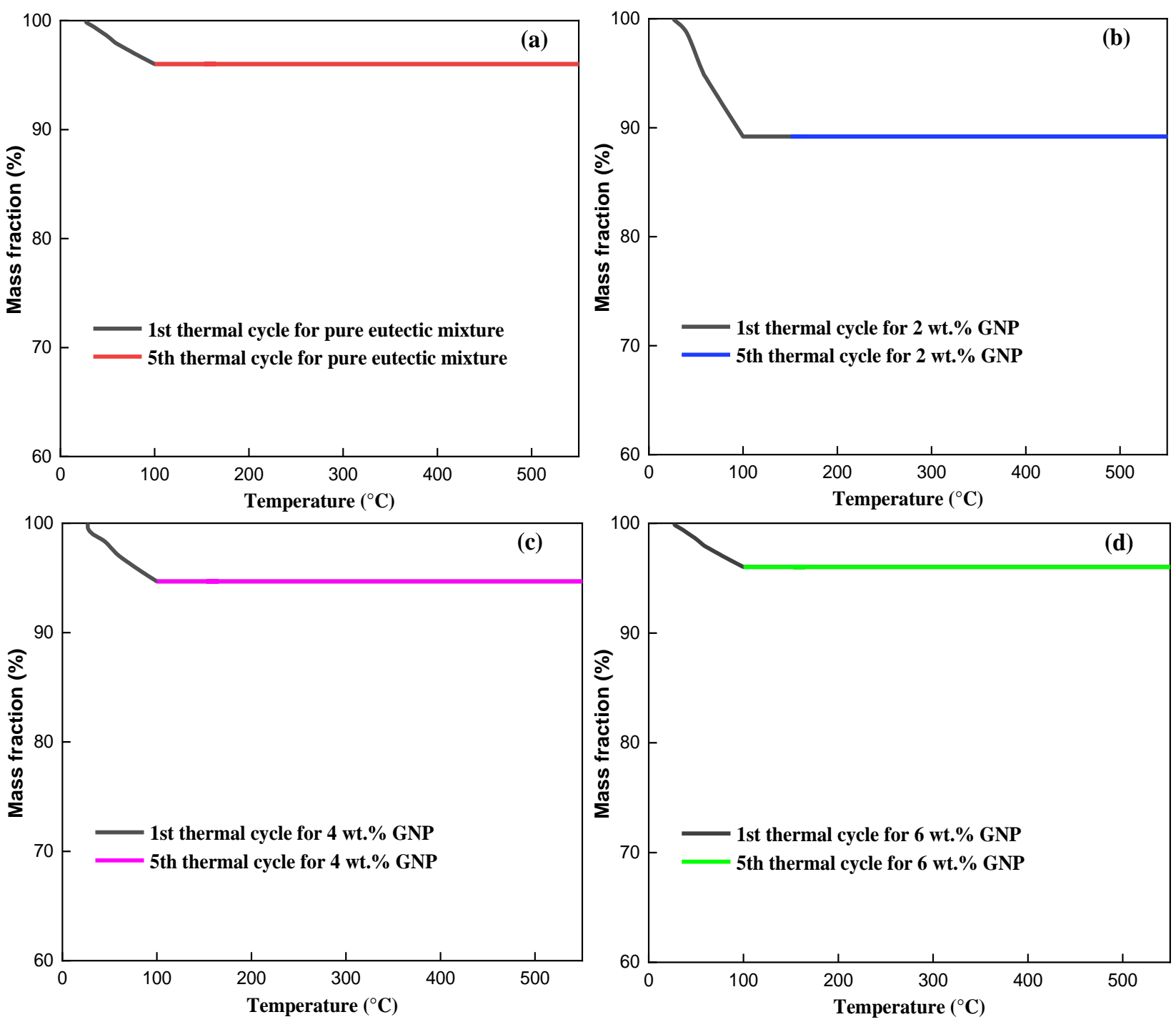

Fig. 5 Variation of mass loss with temperature obtained from thermogravimetric analysis for (a) pure eutectic mixture, (b) $2 \%$ GNP, (c) $4 \%$ GNP, and (d) $6 \%$ GNP-based eutectic mixture.

evaporation. The mass fraction did not vary with temperature for the rest of the thermal cycles $\left(150^{\circ} \mathrm{C}-\right.$ $560^{\circ} \mathrm{C}$ ). Moreover, no mass loss was observed in the melting process. However, for 2 wt.\% GNP-based molten salt, decrement in mass was significant up to $100^{\circ} \mathrm{C}$ which indicates that GNPs can retain moisture content more than that of the pure eutectic mixture which can negatively affect the performance of TES material. With the increment in mass concentration of nanoparticles, a decrement in loss of water vapor was observed from Fig. 5. It indicates that increasing the mass fraction of nanoparticles in base salts induces a mechanism that prohibits graphite nanoparticles from absorbing moisture content. Other than the loss of water, no weight loss in this large temperature range was observed, which also indicates the chemical and thermal stability of all the samples over a wide temperature range.

\section{DISCUSSION}

The drastic enhancement in specific heat capacity of nanocomposites and nanofluids does not align with the theoretical prediction. Several factors can affect the enhancement of $C_{p}$ of pure eutectic mixture after mixing it with nanoparticles. Particle size, shape, and temperature have been reported as the controlling factors of specific heat capacity enhancement [26]. Also, specific heat capacity enhances with decreasing particle size and increasing temperature. To have a closer look at the role of concentration on modifying specific heat capacity, we employed one carbon allotrope with very high potential - graphite nanoparticles. The specific heat capacity of nanoparticles themselves increases with temperature but to account for the enhancement observed at $400{ }^{\circ} \mathrm{C}$ for $6 \mathrm{wt} . \%$ graphite, the specific heat capacity of graphite must be enhanced about $240 \%$ from the value it possesses at $250^{\circ} \mathrm{C}$. So, the increment of temperature contributes to the gain, but the observed enhancement is quite large and cannot be explained solely from temperature increment and associated enhancement of the specific heat capacity of the nanoparticle. A closer look on the morphology of the prepared samples revealed the additional contributing factors for specific heat capacity enhancement.

The homogeneous dispersion of nanoparticles has been considered an important factor to control the thermal properties of the base material. Anomalous increase in specific heat capacity is associated with the formation of a semi-crystalline solid layer on the surface of nanoparticles which in turn depends on the homogeneous dispersion of nanoparticles. Oh et al. reported a TEM image that showed the presence of compressed layers formed on alumina specimens [27]. A molecular 

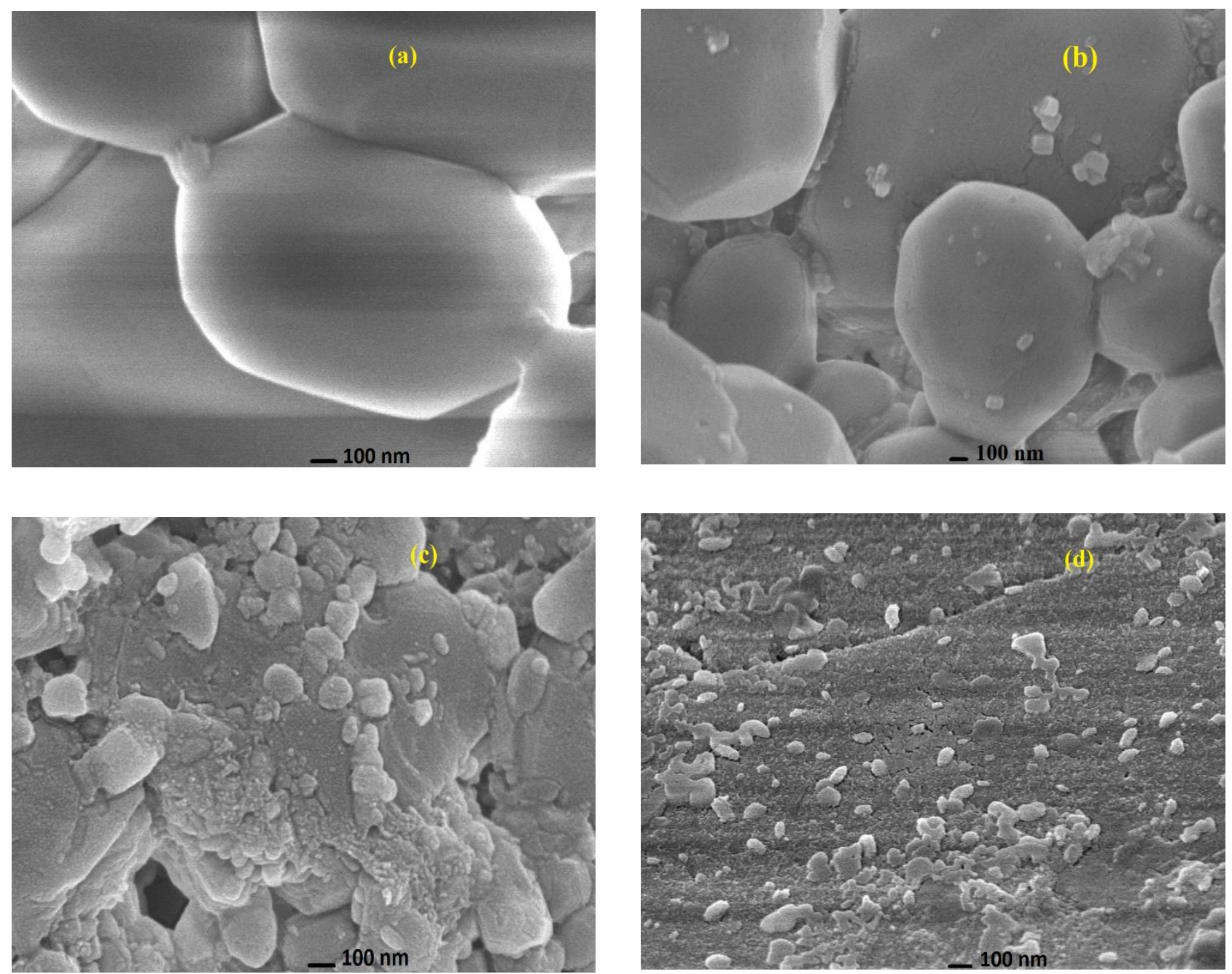

Fig. 6 SEM images of pre-STA samples of (a) pure eutectic mixture, (b) nanocomposite with $2 \%$ GNP, (c) nanocomposite with $4 \%$ GNP, and (d) nanocomposite with $6 \%$ GNP at room temperature.

dynamics (MD) simulation study on the presence of compressed layer on single-walled carbon nanotube (SWCNT) demonstrated that surface charge of nanoparticles plays a role to form a denser layer of salt ions on the SWCNT surface than they remain in the bulk liquid [18]. Electrostatic force plays a vital role to form this nanolayer. However, in the absence of surface charge, Van der Waals force comes in act to form compressed layers on the SWCNT surface. This semi-crystalline layers on the nanoparticle surface form a percolated structure by joining each other. This structure amplifies the heat capacity of molten salt as the dense ion layer possesses higher thermal properties than the bulk fluid.

Fig. 6 shows the SEM images of the prepared samples. The pure eutectic mixture did not show any presence of the percolated network or substructure on the sample surface. The nanocomposite with 2 wt.\% GNPs exhibited well-dispersed nanoparticles and some substructures adjacent to the surface, which has been reported as compressed layers in works of literature [11-12]. But these structures did not form any network connecting each other. The nanocomposite with $4 \mathrm{wt} . \%$ nanoparticle concentration exhibited a higher number of substructures adhered to the surface as well as forming a network among themselves. The compressed layers and network like structures increased with further increment in mass concentration. As can be seen from SEM image, $6 \mathrm{wt} . \%$ GNP-based nanocomposite displayed well dispersed particles, higher number of substructures on the surface of salt mixture, and densely located percolated structure.
Compressed layers do not exist without the presence of nanoparticles and increases with increasing mass concentration. The intermolecular spacing is less in the compressed layer than in bulk liquid. It can be presumed that resulting easy transmission in vibration from closely packed molecules results in higher surface energy- hence, higher specific heat capacity.

Thus, for a given type of nanoparticle, the specific heat capacity depends on the concentration of nanoparticle in the molten salt. Though a further rise in concentration can result in a decrement in specific heat capacity of the molten salt. As stated previously, specific heat capacity depends upon the size, shape, and homogeneous dispersion of nanoparticles, and thus the formation of compressed layers is a surface phenomenon that increases with the increment of available nanoparticle surface. Increasing the mass concentration of homogeneously dispersed nanoparticles offer more surface to form compressed layers but higher mass fraction can result in agglomeration of nanoparticles. Agglomerated particles offer less surface to form compressed layers resulting in lower specific heat capacity. So there exists a threshold concentration for each type of nanoparticles which can be used as a dopant to increase the specific heat capacity of eutectic mixtures. In our study, specific heat capacity enhanced up to 6 wt.\% nanoparticle inclusion but further studies with higher nanoparticle concentration need to be carried out to find out the threshold concentration for graphite nanoparticles, which is a subject of our future study. 
From thermal stability analysis, we can see that increasing mass concentration in nanocomposites resulted in retaining less moisture content in the sample. Graphite can absorb a higher amount of water. However, an assumption can be made here from the analysis of SEM images that the percolated network prohibits water from penetrating through the nanoparticle surface by forming a covered layer on the surface. As a result, a higher amount of percolated network resulted in possessing a lesser amount of moisture content ensuring the low-pressure performance of nanofluids in the TES system.

\section{CONCLUSIONS}

In conclusion, the influence of graphite nanoparticle concentration on the specific heat capacity of the molten salt for three different concentrations $(2,4,6 \mathrm{wt} . \%)$ was examined in our study. Theoretical prediction from thermal equilibrium model was compared with the experimental results. The results are summarized below:

(a) The highest specific heat capacity enhancement was observed in case of 6 wt.\% graphite nanoparticle inclusion in liquid phase which was $25 \%$.

(b) The formation of compressed layers and associated percolated network was confirmed by SEM images and it is a unique structure that can be found in presence of nanoparticles.

(c) $C_{p}$ enhanced with increasing mass concentration of graphite nanoparticles. This means more nanoparticles inclusion ensured more formation of compressed layers for the availability of more nanoparticle surfaces.

(d) Thermal equilibrium model cannot predict the experimental specific heat capacity. This model does not include the effect of particle agglomeration, formation of compressed layers and particle size to predict the $C_{p}$ of nanocomposite, though these are the major controlling factors to enhance the specific heat capacity of nanocomposites.

(e) Presence of water content in the nanocomposites decreases with increasing mass concentration of graphite nanoparticles. Higher number of percolated networks can cover the nanoparticle surface which in turn minimizes the absorption of water by the nanoparticles.

\section{REFERENCES}

[1] I. Dincer, Renewable energy and sustainable development: a crucial review, Renew. Sustain. Energy Rev. 4(2000) 157-175.

[2] N. Navarrete et al., Thermal energy storage of molten salt -based nanofluid containing nano-encapsulated metal alloy phase change materials, Energy 167(2019) 912-920.

[3] H. Zhang, D. Shin, S. Santhanagopalan, microencapsulated binary carbonate salt mixture in silica shell with enhanced effective heat capacity for high temperature latent heat storage, Renew. Energy 134(2019) 1156-1162.

[4] B. Jo, D. Banerjee, Thermal properties measurement of binary carbonate salt mixtures for concentrating solar power plants, J. Renew. Sustain. Energy 7(2015) 033121.
[5] A. Tibodee, T. Thaweechai, W. Sirisaksoontorn, Effect of Size-Controlled Graphene Quantum Dots Combined with $\mathrm{Ni}(\mathrm{OH})_{2}$ for Supercapacitor Application, IEICES 4 (2018) 127-129

[6] L. Qiu et al., A review of recent advances in thermophysical properties at the nanoscale: From solid state to colloids. Phys. Rep. 843(2020) 1-81.

[7] R. M. Sarviya, V. Fuskele, Review on Thermal Conductivity of Nanofluids, Mater. Today Proc. 4(2017) 4022-4031.

[8] H. Younes, G. Christensen, D. Li, H. Hong, A. Al Ghaferi, Thermal Conductivity of Nanofluids: Review, J. Nanofluids 4(2015).

[9] S.-Q. Zhou, R. Ni, Measurement of the specific heat capacity of water-based $\mathrm{Al}_{2} \mathrm{O}_{3}$ nanofluid, Appl. Phys. Lett. 92(2008) 093123.

[10] S. Akilu, A. T. Baheta, K. V. Sharma, M. A. Said, Experimental determination of nanofluid specific heat with $\mathrm{SiO}_{2}$ nanoparticles in different base fluids, 1877(2017) 090001.

[11] D. Shin, D. Banerjee, Enhancement of specific heat capacity of high-temperature silica-nanofluids synthesized in alkali chloride salt eutectics for solar thermal-energy storage applications, Int. J. Heat Mass Transf. 54(2011) 1064-1070.

[12] B. Jo, D. Banerjee, Enhanced Specific Heat Capacity of Molten Salt-Based Carbon Nanotubes Nanomaterials, J. Heat Transf. 137(2015) 091013.

[13] B. Jo, D. Banerjee, Enhanced Specific Heat Capacity of Molten Salts Using Organic Nanoparticles, Heat and Mass Transport Processes, ASMEDC (2011) 1099-1106.

[14] H. Tiznobaik, D. Shin, Enhanced specific heat capacity of high-temperature molten salt-based nanofluids, Int. J. Heat Mass Transf. 57(2013) 542548.

[15] B. Jo, D. Banerjee, Enhanced specific heat capacity of molten salt-based nanomaterials: Effects of nanoparticle dispersion and solvent material, Acta Mater. 75(2014) 80-91.

[16] H. Kim, B. Jo, Anomalous Increase in Specific Heat of Binary Molten Salt-Based Graphite Nanofluids for Thermal Energy Storage, Appl. Sci. 8(2018) 1305.

[17] B. Jo, D. Banerjee, Effect of Dispersion Homogeneity on Specific Heat Capacity Enhancement of Molten Salt Nanomaterials Using Carbon Nanotubes. J. Sol. Energy Eng. 137(2015) 011011.

[18] F. Yuan, Y. L. He, Z. Ma, M. J. Li, Study on the compressed ion layer and the specific heat of the phase change materials doping charged single-walled carbon nanotubes, Energy Procedia 158(2019) 49094914.

[19] B. Jo, D. Banerjee, D. Effect of solvent on specific heat capacity enhancement of binary molten saltbased carbon nanotube nanomaterials for thermal energy storage, Int. J. Therm. Sci. 98(2015) 219-227.

[20] ASTM International Website: http://www.astm.org/cgi-bin/resolver.cgi?E126911( accessed 16.08.2020).

[21] M. A. Islam, K. Thu, B. B. Saha, Specific heat capacity measurement of mangrove and waste palm trunk in raw, carbonized and activated form, IEICES 4 (2018) 151-152. 
[22] J. Buongiorno, Convective Transport in Nanofluids, J. Heat Transf. 128(2006) 240-250.

[23] S. Kline and F. Mcclintock, Describing Uncertainties in Single-Sample Experiments, Mechanical Engineering 75(1953) 3-8.

[24] N. Araki, M. Matsuura, A. Makino, T. Hirata, Y. Kato, Measurement of thermophysical properties of molten salts: Mixtures of alkaline carbonate salts, Int. J. Thermophys. 9(1988) 1071-1080.

[25] D. E. Gray, American Institute of Physics Handbook, Mcgraw-Hill, New York, 1972, pp. 4-106-109

[26] B. X. Wang, L. P. Zhou, X. F. Peng, Surface and Size Effects on the Specific Heat Capacity of Nanoparticles, Int. J. Thermophys. 27(2006) 139-151.

[27] S. H. Oh, Ordered Liquid Aluminum at the Interface with Sapphire. Science 310(2005) 661-663. 